\title{
Functional Recovery and Functional Magnetic Resonance Imaging changes Following Cellular Therapy in a Case of Chronic Complete Spinal Cord Injury
}

\author{
Alok Sharma ${ }^{1}$, Prerna Badhe ${ }^{4}$, Suhasini Pai ${ }^{2 *}$, Hemangi Sane $^{2}$, Samson Nivins ${ }^{2}$, Pooja Kulkarni ${ }^{2}$, Amruta \\ Paranjape ${ }^{2}$, Dhara Mehta $^{3}$ and Nandini Gokulchandran ${ }^{1}$ \\ ${ }^{1}$ Department of Medical Services and Clinical research, Neuro Gen Brain \& Spine Institute, India
}

${ }^{2}$ Department of Research \& Development, Neuro Gen Brain \& Spine Institute, India

${ }^{3}$ Department of Neuro rehabilitation, Neuro Gen Brain \& Spine Institute, India

${ }^{4}$ Department of Regenerative Laboratory Services, Neuro Gen Brain \& Spine Institute, India

Submission: : April 07, 2017; Published: April 27, 2017

*Corresponding author:Suhasini Pai, Department Of Research \& Development, Neuro Gen Brain \& Spine Institute, Stem Asia Hospital and Research Centre, Sector-40, Plot No 19, Palm Beach Road, Seawoods (W), New Mumbai -400706, India, Tel: 91-9920200400; Email: publications@neurogen.in

\begin{abstract}
Functional Magnetic Resonance Imaging (fMRI) is a non-invasive technique to monitor brain activity that provides detailed maps of the brain areas. A number of task-induced fMRI studies have demonstrated rearrangement of cortical activation patterns in the secondary brain areas in SCI patients. Chronic spinal cord injury (SCI) is a devastating disorder afflicting millions across the world. Cellular transplantation is one of the recent strategies to treat chronic complete SCI through its neuroregenerative and neurorestorative property. In this case we administered autologous bone marrow mononuclear cells intrathecally. The follow up assessment was conducted at three and seven months after the cellular therapy. Improvements in gross and fine motor activities, ambulation, bed mobilities, transfers, gait and bladder management were noted over a period of 7 months after the intervention. Spinal Cord Independence Measure (SCIM) improved from 27 to 64/100 and Functional Improvement Measure (FIM) improved from 64 to 83 within seven months of cellular therapy. fMRI showed activation of multiple regions in the sensory and associated areas which was not seen prior to the cellular therapy. No adverse events were reported post cellular therapy. This case study suggests clinical benefits of cellular therapy with multidisciplinary neurorehabilitation especially in chronic SCI. We recommend that its effectiveness should be established in a more comprehensive randomised controlled study for chronic SCI.
\end{abstract}

Keywords: Autologous bone marrow mononuclear cells; Spinal cord injury; Stem cell therapy; Stem cells

\section{Introduction}

Functional Magnetic Resonance Imaging (fMRI) is a noninvasive technique to monitor brain activity that provides detailed maps of the brain areas underlying human mental activities. fMRI estimates blood flow changes in the brain that accompany increases in neural activity,which is then recorded and converted into images of brain activation [1]. fMRI studies have demonstrated rearrangement of cortical activation patterns in the secondary brain areas during task-induced brain activation in spinal cord injury (SCI) patients [2-5]. In humans, studies looking at changes in cortex have been focused on more acute timeframes and have studied multiple spinal cord injury grades $[6,7]$.

Spinal cord injury (SCI) is the most devastating ailment causing permanent loss of sensation and movement in the affected limbs and trunk, bowel/ bladder dysfunction resulting in extreme mental and emotional stress in the patients [8]. The degree of functional loss depends upon the level of injury and on the neurological completeness of the injury [9]. In chronic phase, proliferation of the glial scar is observed along with cyst formation at the necrotic part of the injury [10]. The regeneration and elongation of axon can get blocked by the glial scar which can hinder with functional improvement after the injury [11]. Moreover, intrinsic repair is constrained after SCI as development and maturation neural cells in the central nervous system (CNS) is limited after a certain stage [9]. Standard management approaches in chronic SCI focuses on rehabilitation, pain relief and prevention of secondary complications [11-13]. Therefore it is crucial to find a modality that can stimulate recovery after longstanding injury. Cellular transplantation is one of the recent 
strategies in the treatment of chronic SCI that aims at reducing cell death and promoting regeneration and tissue repair at the site of injury $[11,13]$. In this study, we per 7 formed autologous bone marrow-derived mononuclear cell (BMMNC) transplantation to study the therapeutic effects of cell therapy in chronic SCI. This case study therefore highlights the fMRI changes and explores the clinical effects of transplantation of BMMNCs in chronic SCI.

\section{Case Representation}

A 32-year male met with a car accident in March 2014 during which he sustained a fracture of vertebral body D9 and D10. He underwent decompression with instrumentation and bone grafting on the next day of road traffic accident (RTA) and was hospitalised for a month. He was started on physiotherapy. There was weakness in both lower limbs, loss of sensations below waist level and absence of bowel and bladder control.

On detailed assessment prior to the cellular therapy in October 2015, there was normal sensation up to D9, deep pressure sensation at D10-L1 and no sensation below L1. There was no bowel and bladder sensation or control. Bladder management was through an indwelling catheter. He was hypertonic with grade 1 spasticity in the bilateral hamstrings, right quadriceps, right hip adductors and left glutei and grade 2 spasticity bilateral hip adductors and right glutei. There was tightness in the hip flexors and restriction in right hip flexion with internal rotation. He had involuntary movements with flexor spasm. Brisk reflexes were evident in the lower limbs. Static balance in sitting was fair, whereas dynamic balance was poor. Bed mobilities and transfers to different levels were difficult. He was non-ambulatory. He was partially dependent for activities of daily living.

Functional Independence Measure (FIM) score was found to be 64. On ASIA scale he was at level A. SCIM score was 27/100. MRI dorsal spine suggested fractures of the D9 and D10 vertebral bodies as described with resultant loss of alignment, moderate to severe canal narrowing at this level. Functional Magnetic resonance imaging (fMRI) showed no activation of the sensory or motor cortex on foot movements which is suggestive post traumatic transaction of the cord.

\section{Procedure}

The selection of the patient was based on the World Medical Associations Helsinki declaration [14]. The protocol was reviewed and ethical approval was obtained from Institutional Committee for Stem Cell Research and Therapy (IC-SCRT). The procedure of cellular therapy was explained in detail to the patient and a duly filled informed consent was obtained prior to the therapy.

Before the intervention, the patient underwent a complete evaluation consisting of neurological, psychological and preoperative assessment to assess the preanesthesia fitness. Granulocyte-Colony Stimulating Factor (G-CSF) (300mcg) injections were administered subcutaneously, 72 hours and 24 hours prior to bone marrow aspiration. On the day of transplantation, $100 \mathrm{ml}$ bone marrow was aspirated from the left anterior superior iliac spine under local anesthesia, using bone marrow aspiration needle and was collected in heparinized tubes. The BMMNCs were separated from the aspirate using density gradient method. The purified MNCs were tested for total cell count, viability and CD34+cell content by Fluorescence Activated Cell sorting (FACS). CD34+count was found to be 117cells/uL. The separated cells were then injected intrathecally at the level between L4 and L5. Simultaneous intravenous administration of $1 \mathrm{gm}$ methyl prednisolone in $500 \mathrm{ml}$ of Ringer Lactate solution was carried out to decrease immediate inflammation and to enhance the survival of the injected cells. Total numbers of cells injected were $2.64 \times 107$ with $98 \%$ viability.

Following the transplantation, he underwent multidisciplinary neuro rehabilitation. Physiotherapy consisted of rolling, trunk rotation, assisted bridging, trunk strengthening exercises, push-ups, bed mobility exercises and suspension exercises. It aimed at increasing the trunk control, strength of the preserved muscles and improving balance and gait. Occupational therapy aimed at improving the pelvic control, bed mobility, transfer techniques, lower extremity control and balance. Counselling was provided by a psychologist to cope better with the disease.

The patient was discharged at one-week post transplantation and was advised to continue the rehabilitation at home. The follow up assessment was conducted at three and seven months after the intervention.

Result

Immediately after the cellular therapy, there were no adverse events observed but the clinical course was uneventful. He underwent a multidisciplinary neurorehabilitation regime that was continued for 7 months thereafter.

Within 3 months of cellular therapy, sitting balance improved as he could sit independently on bed for 2 hours. In sitting position, he could perform functional activities like grooming, upper body dressing thereby registering an improvement in his SCIM score from 27 to 58. Improvements were seen in his trunk mobility and mat activities such as sitting up from lying position, transfers from bed to wheelchair. Improvement in locking of the knee was observed. He could ambulate with forearm walker by hopping. A slight improvement was seen in the bladder control. All these improvements were supported by the improved FIM score from 64 to 83.

Over the period of 7 months, the muscle strength in the hip and knee improved with exercises aimed at strengthening of upper limb muscles using suspension therapy with springs and push up bars. The increased muscular strength was mainly seen in the hip adductors and quadriceps, which was evident from the improved balance while sitting and standing and increased ease of transfers at different levels. He could perform push-ups and quadrapeds which were difficult earlier. Superficial fine touch 


\section{Current Trends in Clinical \& Medical Imaging}

could be felt at the D10 level. All these improvements led to a further increase in SCIM from 58 to 64 and FIM from 83 to 89. Rest of his improvements have been maintained. fMRI investigation with movements of the right foot revealed activation in post central gyrus, sensory motor area and few associated areas in the left hemisphere (Figure 1).

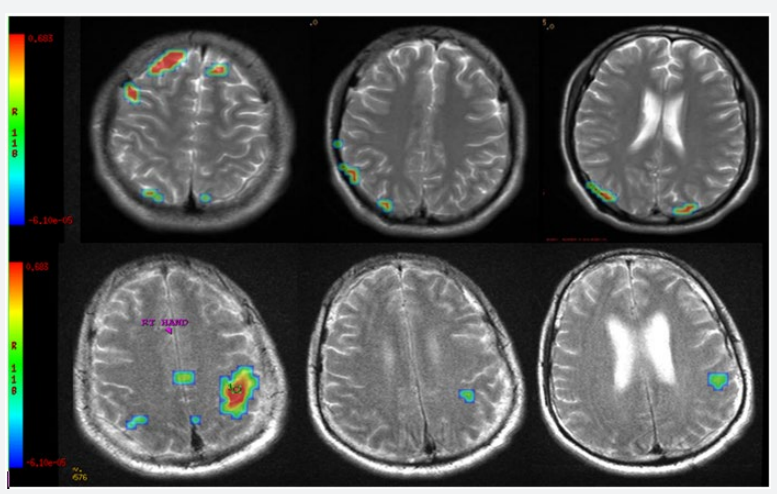

Figure 1: Pre cellular therapy-Representation of T-2 Axial weighted sections showing activation of the right superior frontal gyrus, Right middle frontal gyrus with few activation of associated regions by performing right foot movements. Post cellular therapy: The analysis for the right foot movements demonstrating the activation in contralateral T2- weighted on Axial sections. The significant increase in activation isobserved in post cell therapy showing activation in post central gyrus and sensory motor area of the left hemisphere with few associated area and supramarginal.

\section{Discussion}

Spinal cord injury (SCI) is a devastating disorder afflicting millions across the world with no existing effective standard cure [15]. Recovery from injury is restricted due to the confined ability of the injured nervous system to replace disrupted myelin, and re-establish functional neural connections [16]. So far, medical treatment and rehabilitation have focused on preventing complications and maximizing residual functional capacities in acute spinal cord injuries [17]. But there are no such treatment modalities for chronic cases. Cellular therapy has been gaining importance as a therapeutic approach to treat SCI through its neuroregenerative and neurorestorative property. Stem cells enhance angiogenesis, differentiate into neuronal and oligodendrocytes cells and induce neuro protective effect by secreting certain cytokines [18-20]. Stem cell derived exosomes are proposed to have a regulatory function in synaptic plasticity and myelin membrane biogenesis [21]. All these mechanismshave shown to improve the functional and symptomatic outcomes in patients with chronic cervical and thoracolumbar spinal cord injuries [22,23].

In this case we administered bone marrow mononuclear cells intrathecally as cells through this path are well tolerated and migrate efficiently to the contused spinal cord tissues and exert a significant beneficial effect [24]. The patient also underwent a multidisciplinary rehabilitation regime post cellular therapy.
It is well established that exercises along with cellular therapy promotes the recovery of neurological deficits in spinal cord trauma through release of neurotrophic factors that stimulate neuronal growth [25].

Functional magnetic resonance imaging (fMRI) is a powerful tool for mapping neuronal function and depicts patterns of activation for attempted movement [26]. fMRI of the spinal cord has various challenges. First challenge arises from the fact that the spinal cord is contained within the vertebral column and hence is relatively inaccessible. Second is the difference in the magnetic susceptibilities between the bone, cartilage, tissues and surgical implants around the spinal cord that might cause distortions in the imaging [27]. Hence in this study, fMRI of the brain was studied. In few studies done earlier, serial fMRI scans have shown that the progression of structural changes at the brain and spinal levels occurs with a specific spatial and temporal pattern. These changes are induced by retrograde degeneration of the motor fibres along the corticospinal tract resulting in the shrinkage of cytoplasm of the cortico spinal neurons $[27,28]$. Cross-sectional images of the spinal cord and brain in chronic spinal cord injury patients have shown correlations between structural changes and neuronal deficits. The preservation of structural integrity, at the spinal and brain levels would therefore decide the neurological and functional improvements after intervention [29,30].

Experimental evidence suggests that axonal remodeling and plasticity occurs not only within the spinal cord below and above the lesion but also within the brain both subcortically and cortically, despite the detrimental impact of the trauma [31,32]. Improved gait, slight improvement in the bladder sensations and superficial fine touch along with enhanced functional status, as recorded in the SCIM scale in this case suggests improvement in neuronal function. The improvement in transfers, balance and lower limb control could be due to strengthening of muscles. Through the neuroprotective and regenerative property of cellular therapy, repair and re-organisation at the site of injuryand adjacent areas of the brainmay have led to these improvements in this case.

\section{Conclusion}

fMRI can be viewed as an impressive tool to monitor the effects of cellular therapy, although a systematic protocol is required. In this case study suggests functional recovery after cellular therapy which was not achieved with standard physiotherapy and surgical intervention. The neurorestorative property of BMMNC could be responsible for functional improvements seen in the patient, even at the chronic stage. This case study demonstrates clinical benefits of combination of cellular therapy and rehabilitation especially in chronic SCI. We recommend that its effectiveness should be established in a more comprehensive randomised control study for chronic SCI.

References 


\section{Current Trends in Clinical \& Medical Imaging}

1. Stroman PW (2005) Magnetic Resonance Imaging of Neuronal Function in the Spinal Cord: Spinal fMRI. Clin Med Res 3(3): 146-156.

2. Liverman CT, Altevogt BM, Joy JE (2005) Spinal Cord Injury: Progress, Promise and Priorities. National Academies Press, Washington DC, USA.

3. Lundell H, Christensen MS, Barthélemy D, Willerslev-Olsen M, BieringSørensen F, et al. (2011) Cerebral activation is correlated to regional atrophy of the spinal cord and functional motor disability in spinal cord injured individuals. NeuroImage 54(2): 1254-1261.

4. Hotz-Boendermaker S, Funk M, Summers P, Brugger P, HeppReymond MC, et al. (2008) Preservation of motor programs in paraplegics as demonstrated by attempted and imagined foot movements. Neuroimage 39(1): 383-394.

5. Jurkiewicz MT, Crawley AP, Verrier MC, Fehlings MG, Mikulis DJ (2006) somatosensory cortical atrophy after spinal cord injury: a voxel-based morphometry study. Neurology 66(5): 762-764.

6. Choe AS, Belegu V, Yoshida S, Joel S, Sadowsky CL, et al. (2013) Extensive neurological recovery from a complete spinal cord injury: a case report and hypothesis on the role of cortical plasticity. Front Hum Neurosci $7: 290$.

7. Min YS, Park JW, Jin SU, Jang KE, Nam HU, et al. (2015) Alteration of Resting-State Brain Sensorimotor Connectivity following Spinal Cord Injury: A Resting-State Functional Magnetic Resonance Imaging Study. J Neurotrauma 32(18):1422-1427

8. McDonald JW, Sadowsky C (2002) Spinal-cord injury. The Lancet 359(9304): 417-425.

9. Shibuya S, Yamamoto T, Itano T (2009) Glial and axonal regeneration following spinal cord injury. Cell Adh Migr 3(1): 99-106.

10. Bovolenta P, Wandosell F, Nieto-Sampedro M (1992) CNS glial scar tissue: a source of molecules which inhibit central neurite outgrowth. Prog Brain Res 94: 367-379.

11. Baptiste DC, Fehlings MG (2007) Update on the treatment of spinal cord injury. Prog Brain Res 161: 217-233.

12. Hernández J, Torres-Espín A, Navarro X (2011) Adult stem cell transplants for spinal cord injury repair: current state in preclinical research. Curr Stem Cell Res Ther 6(3): 273-287.

13. Tator CH, Hashimoto R, Raich A, Norvell D, Fehlings MG, et al. (2012) Translational potential of preclinical trials of neuroprotection through pharmacotherapy for spinal cord injury. J Neurosurg Spine 17(1 Suppl): 157-229.

14. Carlson RV, Boyd KM, Webb DJ (2004) The revision of the Declaration of Helsinki: Past, present and future. Br J Clin Pharmacol 57(6): 695713

15. Freund P, Weiskopf N, Ashburner J, Wolf K, Sutter R, et al. (2013) MRI investigation of the sensorimotor cortex and the corticospinal tract after acute spinal cord injury: a prospective longitudinal study. Lancet Neurol 12(9): 873-881.

16. Rahimi-Movaghar V (2005) Efficacy of Surgical Decompression in the Setting of Complete Thoracic Spinal Cord Injury. J Spinal Cord Med
28(5): 415-420

17. Anderson KD (2004) Targeting recovery: Priorities of the spinal cordinjured population. J Neurotrauma 21: 1371-1383.

18. Zhang N, Yin Y, Xu SJ, Wu YP, Chen WS, et al. (2012). Inflammation \& apoptosis in spinal cord injury. Indian J Med Res 35(3): 287-296.

19. Mehler MF, Rozental R, Dougherty M, Spray DC, Kessler JA (1993) Cytokine regulation of neuronal differentiation of hippocampal progenitor cells. Nature 362(6415): 62-65.

20. Ogawa Y, Sawamoto K, Miyata T, Miyao S, Watanabe M, et al. (2002) Transplantation of in vitro-expanded fetal neural progenitor cells results in neurogenesis and functional recovery after spinal cord contusion injury in adult rats. J Neurosci Res 69(6): 925-33.

21. Aryani A, Denecke B (216) Exosomes as a nanodelivery system: a key to the future of neuromedicine? Mol Neurobiol 53(2): 818-834.

22. Sharma A, Gokulchandran N, Sane H, Badhe P, Kulkarni P, et al. (2013) Detailed analysis of the clinical effects of cell therapy for thoracolumbar spinal cord injury: an original study. Journal of Neurorestoratology 1 : 13-22.

23. Alok Sharma, Hemangi Sane, Nandini Gokulchandran, Pooja Kulkarni et al. (2013) Role of autologous bone marrow mononuclear cells in chronic cervical spinal cord injury-a long term follow-up study. Journal of Neurological Disorders 1(4).

24. Bakshi A, Barshinger AL, Swanger SA, Madhavani V, Shumsky JS, et al. (2006) Lumbar puncture delivery of bone marrow stromal cells in spinal cord contusion: a novel method for minimally invasive cell transplantation. J Neurotrauma 23(1): 55-65.

25. Carvalho KA1, Cunha RC, Vialle EN, Osiecki R, Moreira GH et al. (2008) Functional outcome of bone marrow stem cells (CD45(+)/CD34(-)) after cell therapy in acute spinal cord injury: in exercise training and in sedentary rats. Transplant Proc 40: 847-849.

26. Stroman PW (2005) Magnetic Resonance Imaging of Neuronal Function in the Spinal Cord: Spinal fMRI. Clin Med Res 3(3): 146-156.

27. Hains BC, Black JA, Waxman SG (2003) Primary cortical motor neurons undergo apoptosis after axotomizing spinal cord injury. J Comp Neurol 462(3): 328-341.

28. Feringa ER, Vahlsing HL, Smith BE (1983) Retrograde transport in corticospinal neurons after spinal cord transection. Neurology 33(4): 478-482.

29. Freund P, Curt A, Friston K, Thompson A (2013) Tracking changes following spinal cord injury: insights from neuroimaging. Neuroscientist 19(2): 116-128.

30. Freund P, Weiskopf N, Ashburner J, Wolf K, Sutter Ret al. (213) MRI investigation of the sensorimotor cortex and the corticospinal tract after acute spinal cord injury: a prospective longitudinal study. Lancet Neurolog 12(9): 873-881.

31. Jacobs KM, Donoghue JP (1991) Reshaping the cortical motor map by unmasking latent intracortical connections. Science 251(4996):944947. 
Your next submission with Juniper Publishers will reach you the below assets

- Quality Editorial service

- Swift Peer Review

- Reprints availability

- E-prints Service

- Manuscript Podcast for convenient understanding

- Global attainment for your research

- Manuscript accessibility in different formats

( Pdf, E-pub, Full Text, Audio)

- Unceasing customer service

Track the below URL for one-step submission https://juniperpublishers.com/online-submission.php 